\title{
Metodologias para a criação de jogos educacionais: um Mapeamento Sistemático da Literatura
}

\author{
Rafaela Melo ${ }^{1}$, Marcela Pessoa ${ }^{1,2}$, Fernanda Pires ${ }^{1,2}$ \\ Polianny Lima ${ }^{1}$, David B. F. Oliveira ${ }^{2}$
}

${ }^{1}$ Escola Superior de Tecnologia - Universidade do Estado do Amazonas (UEA) ThinkTEd Lab - Pesquisa e Desenvolvimento em Tecnologias Educacionais Manaus - AM - Brasil

${ }^{2}$ Programa de Pós-Graduação em Informática Instituto de Computação - Universidade Federal do Amazonas (UFAM) Manaus - AM - Brasil

\{rmf.lic16,fpires,palima,msppessoa\}@uea.edu.br, davideicomp.ufam.edu.br

\begin{abstract}
Researchers point out the potential of games in the learning process, and over the years different methodologies for creating such games have emerged. Given this scenario, this paper presents a systematic literature mapping that aims to identify the advantages of applying theories and learning criteria in the educational games development process. The searches performed in the databases (Scopus, IEEE, ACM, and Web Of Science) resulted in the selection of 10 articles, based on the acceptance criteria defined in the review protocol. The research results pointed out that the use of theoretical aspects in the process of developing educational games helps in better learning results.
\end{abstract}

Resumo. Pesquisadores apontam o potencial dos jogos no processo de aprendizagem e, ao longo dos anos, surgiram diferentes metodologias para desenvolvê-los. Diante desse cenário, o presente artigo apresenta um mapeamento sistemático da literatura que tem por objetivo identificar as vantagens de aplicar teorias e critérios de aprendizagem no processo de desenvolvimento de jogos educacionais. As buscas realizadas nas bases de dados (Scopus, IEEE, ACM e Web of Science) resultaram na seleção de dez artigos, a partir dos critérios de aceitação definidos no protocolo de revisão. Os resultados da pesquisa apontaram que a utilização de aspectos teóricos no processo de desenvolvimento de jogos educacionais auxilia em melhores resultados de aprendizagem.

\section{Introdução}

Jogos digitais têm a sua origem em 1962 com o surgimento do Space War. A partir de então, eles se tornaram parte da cultura, influenciando aspectos sociais, políticos, econômicos e tecnológicos [Newman 2012]. Esse cenário despertou o interesse de pesquisadores de diversas áreas (design, ciência da computação, literatura) e, atualmente, um dos principais campos em que os jogos são aplicados é a educação [Hsiao 2007]. Pesquisas apontam que o ato de jogar envolve atividades de aprendizagem mais desafiadoras do que uma tarefa escolar, por exemplo [Kattenbelt and Raessens 2003].

Aspectos como ambientes atraentes e interativos chamam a atenção do jogador propiciando desafios que elevam o nível de habilidade requerida a cada etapa 
[Balasubramanian and Wilson 2006]. Além da motivação, os jogos educacionais proporcionam benefícios como: facilitador da aprendizagem, desenvolvimento de habilidades cognitivas, aprendizagem por descoberta, experiências de novas identidades, socialização e coordenação motora [Gros 2003, Balasubramanian and Wilson 2006, Hsiao 2007, Savi and Ulbricht 2008]. Embora discuta-se o benefício dos jogos para a aprendizagem, existem poucas evidências de que eles são capazes de produzir resultados educacionais válidos e duradouros, ou que sejam melhores que outras formas de aprendizagem [Linehan et al. 2011]. Então, para que os resultados se caracterizem como válidos, é preciso reaver os processos de desenvolvimento de forma a manter o equilíbrio entre a aprendizagem e o entretenimento dentro do jogo, analisando quais estruturas têm potencial para desenvolver aspectos cognitivos [Gros 2003].

Diante disso, este trabalho tem como objetivo identificar as metodologias de desenvolvimento de jogos educacionais e a utilização de teorias/critérios de aprendizagem no processo de desenvolvimento, por meio de um Mapeamento Sistemático da Literatura (MSL). O artigo está organizado como segue: a Seção 2 apresenta a metodologia empregada para o MSL; a Seção 3 trata da discussão dos artigos selecionados, e por último, na Seção 4 estão as considerações finais.

\section{Protocolo do Mapeamento Sistemático da Literatura (MSL)}

Nesta pesquisa foi utilizado o método de Mapeamento Sistemático da Literatura (MSL), onde identificam-se os estudos mais relevantes a partir de uma temática ou de uma questão de pesquisa [Kitchenham and Charters 2007], conforme protocolo descrito a seguir.

\subsection{Questões de pesquisa}

A primeira fase do protocolo é o planejamento, onde são definidos os objetivos do mapeamento sistemático. Por meio deste MSL, pretende-se identificar as vantagens da utilização de critérios de aprendizagem no processo de desenvolvimento de jogos educacionais. Para isso, foram elaboradas as seguintes questões de pesquisa:

\section{Questão Principal:}

- QP1: A utilização de critérios de aprendizagem/teorias de aprendizagem para o processo de desenvolvimento de jogos educacionais ajuda a melhorar o desempenho dos estudantes? Em caso positivo, como é medido o desempenho?

\section{Questões Secundárias:}

- QP1.1: Quais as metodologias utilizadas para a criação de jogos educacionais?

- QP1.2: Quais os critérios de aprendizagem/teorias de aprendizagem utilizados no processo de desenvolvimento dos jogos?

\subsection{Método da pesquisa}

O método PICO (Population, Intervention, Comparison, Outcome) [Petersen et al. 2015] foi utilizado na pesquisa para estruturar o mapeamento, identificando a população (P), a intervenção (I), a comparação (C) e o resultado (O), conforme descritos a seguir:

- Population (P): trabalhos publicados em conferências ou periódicos apresentando o processo de desenvolvimento de jogos educacionais; 
- Intervention (I): utilização de modelos/técnicas/métodos para criação de jogos educacionais e aplicação de teorias/critérios de aprendizagem no processo;

- Comparison $(\boldsymbol{C})$ : não se aplica, visto que o objetivo é caracterizar os estudos;

- Outcome $(\boldsymbol{O})$ : trabalhos que apresentam uma metodologia de desenvolvimento e consideram critérios educacionais para a criação de jogos educacionais.

\subsection{String de busca}

Para responder as questões de pesquisa propostas, a string de busca formou-se a partir de quatro conjuntos de palavras, seguindo o método PICO, como pode ser visto na Tabela 1. As palavras de cada categoria (jogos, metodologia, teoria de aprendizagem e resultado esperado) são conectadas com OR e as categorias com AND.

Tabela 1. Conjunto de Palavras.

\begin{tabular}{|l|l|l|}
\hline \multicolumn{1}{|c|}{ Tipo } & \multicolumn{1}{|c|}{ Categoria } & \multicolumn{1}{c|}{ Palavras } \\
\hline População & Jogos & $\begin{array}{l}\text { game, educational game, serious game, learning game, } \\
\text { cognitive game }\end{array}$ \\
\hline Intervenção & Metodologia & $\begin{array}{l}\text { process, methodology, design, game design, metric, ins- } \\
\text { tructional design, approach }\end{array}$ \\
\cline { 2 - 3 } & Teoria de aprendizagem & $\begin{array}{l}\text { learning theory, constructionism, constructivism, genetic } \\
\text { epistemology, zone of proximal development, behaviou- } \\
\text { rism }\end{array}$ \\
\hline Resultado & Resultado esperado & $\begin{array}{l}\text { learning, effective, advantageous, advantage, potential, } \\
\text { benefit, improvement, motivation, engagement, effici- } \\
\text { ency, performance, dedication, satisfaction, improve- } \\
\text { ment, approval rate, enhance }\end{array}$ \\
\hline
\end{tabular}

Para validar a string de busca, foi selecionado um conjunto de artigos de controle, com cinco artigos considerados relevantes pelos pesquisadores e que ajudavam a responder as questões de pesquisa. A consulta foi considerada satisfatória quando todos os artigos presentes no grupo de controle foram retornados pela string.

\subsection{Bases de busca e critérios de inclusão e exclusão}

Foram consultadas as bases de dados Scopus ${ }^{1}, \mathrm{IEEE}^{2}, \mathrm{ACM}^{3}$ e Web of Science ${ }^{4}$. Os critérios de inclusão (CI) e exclusão (CE) foram definidos como segue:

Critérios de Inclusão: i) $\mathrm{O}$ artigo deve discorrer sobre o processo de desenvolvimento de jogos educacionais; ii) $\mathrm{O}$ artigo deve discorrer sobre os critérios de aprendizagem aplicados no processo de desenvolvimento do jogo.

Critérios de Exclusão: i) Não atender aos critérios de inclusão; ii) $\mathrm{O}$ artigo não ser um artigo completo (considerando para isso no mínimo 5 páginas); iii) A versão completa do artigo não estar disponível entre as fontes selecionadas; iv) O idioma do artigo não ser inglês; v) $\mathrm{O}$ artigo estar duplicado; vi) Ser livro ou capítulo de livro, tese ou

\footnotetext{
${ }^{1}$ https://www.scopus.com

${ }^{2} \mathrm{https} / / /$ ieeexplore.ieee.org

${ }^{3}$ https://dl.acm.org/

${ }^{4}$ https://webofknowledge.com/
} 
dissertação; vii) O estudo não ser um estudo primário (sumário de anais de eventos científicos, descrição de um curso, editorial, resumo de palestra, workshop ou tutorial, mapeamentos sistemáticos da literatura ou mesmo estudos apresentando revisões informais da literatura) e viii) $\mathrm{O}$ estudo ser uma versão mais antiga de outro estudo já considerado.

\section{Resultados}

Na tentativa de reduzir o viés de um único pesquisador, este mapeamento sistemático foi desenvolvido por quatro pesquisadores. A estratégia de divisão de atividades foi inspirada em Oliveira et al. (2017), onde o protocolo de revisão e a estratégia de busca foram especificados por dois pesquisadores e os demais definiram os critérios de inclusão e exclusão. Para avaliar a confiança na seleção dos artigos, dois pesquisadores selecionaram, individualmente, uma amostra aleatória de vinte trabalhos, aplicando os critérios de inclusão. Após a seleção, os pesquisadores analisaram os trabalhos com base no título, resumo e palavras-chave. As classificações foram comparadas e foi utilizado o teste estatístico Kappa [Cohen 1960] para avaliar a confiança na classificação. O teste indicou que os pesquisadores convergiram estatisticamente (Kappa $=0,8$ ) na seleção dos artigos, aumentando a confiança e diminuindo o viés do pesquisador na inclusão dos artigos.

\subsection{Base de busca}

A string resultante da Tabela 1 foi rodada nas quatro bases de busca. A Figura 1 (a) apresenta a quantidade de artigos retornados por base no primeiro filtro. Dos 1009 artigos, 650 são da Scopus, representando 64\% dos trabalhos. Após o último filtro restaram dez trabalhos, oito retornados pela Scopus e dois pela Web of Science (Figura 1 (b)). A Tabela 2 apresenta os artigos resultantes da MSL, seguindo ordem cronológica de publicação.

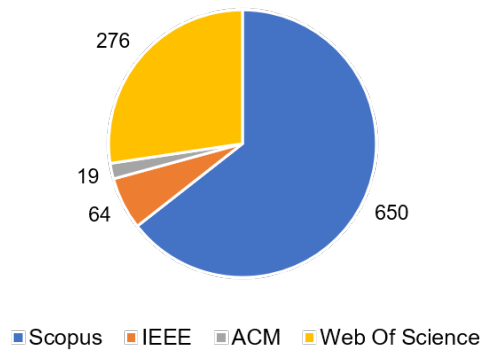

(a)

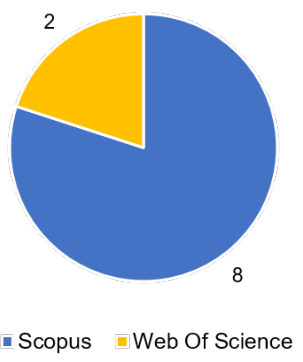

(b)

Figura 1. (a) Primeira Busca, (b) Último Filtro.

Tabela 2. Artigos Selecionados.

\begin{tabular}{l|l|l}
\hline Referência & Referência & Referência \\
\hline [Ahmad et al. 2012] & [Nikitakos et al. 2017] & [Fonseca et al. 2018] \\
$\begin{array}{l}\text { Crow and Parsons 2015] } \\
\text { [Bul et al. 2015] }\end{array}$ & [Sockolow et al. 2018] & [Mohd Rashid et al. 2019] \\
[Piki et al. 2016] & & [Ros et al. 2020] \\
\hline
\end{tabular}

Dada a importância da discussão sobre ameaças à validade [Petersen et al. 2015] no processo de MSL, no que diz respeito à validade teórica, para minimizar o viés do 
IX Congresso Brasileiro de Informática na Educação (CBIE 2020)

Anais do XXXI Simpósio Brasileiro de Informática na Educação (SBIE 2020)

pesquisador, este trabalho utilizou um conjunto de cinco artigos de controle (em negrito na Tabela 2) e o teste estatístico de Kappa [Cohen 1960].

\subsection{QP1.1: Quais as metodologias utilizadas para a criação de jogos educacionais?}

Ahmad et al. (2012) adotaram a prototipagem descartável (throw-away) como metodologia de desenvolvimento, contendo as fases: planejamento, análise, design, implementação e sistema (finalização). Em Crow e Parsons (2015), o jogo foi projetado através de ciclos iterativos de desenvolvimento, teste e avaliação, onde cada etapa de teste forneceu base para a iteração seguinte. Por meio da colaboração interdisciplinar entre profissionais de saúde, pais de filhos com ADHD (attention deficit/hyperactivity disorder), designers, especialistas em comportamento, pesquisadores e desenvolvedores, o jogo proposto em Bul et al. (2015) teve o seguinte processo de desenvolvimento e avaliação: (1) definição dos objetivos de aprendizagem; (2) sessões de brainstorm; (3) criação de protótipos; (4) fase iterativa com avaliação, refinamento e testes de usabilidade; (5) primeiro protótipo; e, (6) estudo piloto. Piki et al. (2016) adaptaram uma metodologia de prototipagem de sistemas combinada com o desenvolvimento de sistemas instrucionais e definiram as seguintes etapas de desenvolvimento, em um ciclo iterativo: planejamento, teoria, análise, design, implementação, avaliação e jogo educacional (entrega do sistema). Um framework para desenvolvimento de jogos educacionais é apresentado em Nikitakos et al. (2017), com as seguintes etapas: análise, design e produção (protótipo, versões beta e gold).

Sockolow et al. (2018) abordaram dez características de game design: referencial teórico, definição do público-alvo (idade, sexo), definição do alvo (local onde o jogo será aplicado), elicitação de necessidades (envolvendo o público escolhido), análise de requisitos (o que foi capturado na elicitação), definição das características do jogo (história, personagens, cenários), escolha do método de disseminação, definição dos resultados esperados, definição das métricas de avaliação e avaliação. Em Zhou et al. (2018), os autores propõem um modelo de desenvolvimento de jogos que apoia do design à avaliação, levando em consideração aspectos técnicos e pedagógicos e possui três etapas principais: orientação de design, processo de jogo e dimensões de avaliação. A metodologia de desenvolvimento de Fonseca et al. (2018) considera três aspectos: design, implementação e teste (durante a codificação). O modelo ADDIE (Analysis, Design, Development, Implementation, and Evaluation) foi utilizado em Mohd Rashid et al. (2019) e possui cinco fases distintas: análise, design, desenvolvimento, implementação e avaliação. O processo de desenvolvimento abordado em Ros et al. (2020) contém cinco etapas: descrição do contexto educacional, seleção do framework de desenvolvimento, descrição da narrativa e das cenas, definição das metáforas de aprendizado e validação; é um processo cíclico que possibilita aumentar a qualidade do jogo.

Observa-se que nenhum dos artigos selecionados usou a mesma metodologia de desenvolvimento, ou seja, há uma falta de padronização nos processos de criação de jogos. Alguns autores defendem a importância de cada etapa do processo, enquanto outros apenas citam essas etapas e não descrevem como foram realizadas.

\subsection{QP1.2: Quais os critérios de aprendizagem/teorias de aprendizagem utilizados no processo de desenvolvimento dos jogos?}

Um jogo para tradução de idiomas é proposto em Ahmad et al. (2012). Os autores aplicaram as seguintes teorias de aprendizagem: behaviorismo, onde as crianças podem jogar 
repetidas vezes para passar de nível; cognitivismo, para apoiar o desenvolvimento préoperacional por meio do desenvolvimento de imagens mentais; e, construtivismo, para ajudar os alunos a criarem suas próprias experiências. Para desenvolver um jogo sobre o idioma Maori, Crow e Parsons (2015) investigaram métodos de aprendizado e identificaram uma abordagem semelhante às possíveis estruturas de um mundo de jogo. Eles se basearam na técnica The Silent Way [Gattegno 2010] onde o aprendizado é facilitado por meio de descoberta, criação, acompanhamento de objetos físicos e solução de problemas que envolvem os objetivos de aprendizagem.

Bul et al. (2015) propõem um jogo sério para crianças com ADHD, cujos objetivos de aprendizagem eram promover o uso de estratégias sobre o cotidiano (organização, planejamento, habilidades pró-sociais). Para isso, os autores se basearam nas seguintes teorias psicológicas: modelo de auto-regulação, teoria social cognitiva e teorias de aprendizagem que defendem que as pessoas aprendem através de comportamentos e reforço positivo. Para criar três jogos educacionais para crianças com deficiência intelectual, em Piki et al. (2016), os autores utilizaram aspectos do modelo ARCS (atenção, relevância, confiança e satisfação) e da teoria do engajamento como base para projetar jogos educacionais pelos quais crianças com deficiência intelectual podem aprender brincando.

Em Nikitakos et al. (2017), os autores defendem que para produzir jogos que combinem entretenimento com aprendizagem é necessário adotar uma estratégia instrucional, que envolva desenvolvedores e educadores especialistas no assunto. Na fase de análise são definidas metas e habilidades de aprendizagem, de modo que os especialistas são responsáveis por avaliar as necessidades e definir os pré-requisitos de aprendizagem e os resultados esperados, e também identificar a teoria de aprendizagem que mais se adequa ao contexto. Já os desenvolvedores precisam combinar os objetivos de aprendizagem traçados com os objetivos de entretenimento. Atraveś do framework proposto, eles desenvolveram um jogo para estudantes da indústria marítima. Em Sockolow et al. (2018) é proposto um jogo sério na área da saúde com a temática do comportamento de risco sexual (SRB - sexual risk behavior) em adolescentes, os autores se basearam em dois referenciais teóricos de acordo com o contexto do jogo, são eles: modelo transteórico de mudança de comportamento, que fornece estratégias para a pessoa adotar um comportamento mais saudável; e a abordagem de atendimento informado ao trauma, que auxilia no desenvolvimento de habilidades relacionadas ao trauma.

No modelo proposto por Zhou et al. (2018) para o desenvolvimento de um jogo sobre classificação de lixo, o construtivismo está subjacente, propondo contextos e atividades que auxiliem na construção de novos conhecimentos. Para orientar o design do jogo, alguns requisitos de aprendizagem são definidos: objetivos, estilos, tarefas e resultados esperados; sendo que os objetivos são baseados na taxonomia de Bloom. Para motivar alunos do ensino médio a seguir carreira nas áreas de STEM (ciências, tecnologia, engenharia e matemática), em Fonseca et al. (2018) é criado um jogo educacional sobre nanotecnologia baseado nas teorias do construtivismo e da mentalidade. Em Mohd Rashid et al. (2019), para criar um jogo com foco no aprendizado de scrips Jawi, os autores se basearam no construtivismo e na GBL - game based learning (aprendizagem baseada em jogos). Um dos aspectos construtivistas abordados no jogo é a mecânica de arrastar e soltar objetos, ajudando a incorporar novas informações e utilizando conhecimentos prévios para resolver os problemas propostos. A GBL é defendida pelo fato de 
auxiliar no desenvolvimento de habilidades de raciocínio. Um jogo sobre segurança cibernética é proposto em Ros et al. (2020), onde os autores traçaram resultados esperados de aprendizagem, considerando princípios como conhecimento prévio e motivação. Além disso, baseou-se nos preceitos da teoria do construtivismo, onde a perspectiva de mundo é construída a partir de experiências individuais. Para que o jogador aprenda a lidar com o universo de jogo, personagens, movimentos e eventos é necessário que exista um ambiente dinâmico. Nesse sentido, considerou-se que o jogo tem uma dupla dimensão de aprendizagem: regras do jogo (obter regras experimentando o jogo e aplicando-as nas demais situações) e jogabilidade (aprender por hipótese e construção de acordo com os mecanismos de resposta oferecidos no jogo).

A Tabela 3 apresenta as teorias utilizadas nos trabalhos selecionados. Nota-se que alguns autores utilizaram mais de uma teoria e que a mais utilizada foi o construtivismo.

Tabela 3. Relação de Teorias e Trabalhos.

\begin{tabular}{|l|l|}
\hline Teoria & Trabalho \\
\hline Construtivismo & $\begin{array}{l}{[\text { Ros et al. 2020], [Mohd Rashid et al. 2019], }} \\
\text { [Zhou et al. 2018], } \\
\text { [Ahmad et al. 2012] }\end{array}$ \\
\hline Game based learning \\
\hline Teoria da mentalidade 2018$],$ \\
\hline Não informa & [Mohd Rashid et al. 2019] \\
\hline Modelo de auto-regulacão e teoria social cognitiva & {$[$ Fonseca et al. 2018] } \\
\hline Técnica The Silent Way & {$[$ Bul et al. 2015] } \\
\hline Behaviorismo e Cognitivismo & {$[$ Crow and Parsons 2015] } \\
\hline $\begin{array}{l}\text { Modelo transteórico de mudança de comporta- } \\
\text { mento e abordagem de atendimento informado ao } \\
\text { trauma }\end{array}$ & {$[$ Ahmad et al. 2012] } \\
\hline Modelo ARCS e teoria do engajamento & {$[$ Sockolow et al. 2018] } \\
\hline
\end{tabular}

\subsection{QP1: A utilização de critérios/teorias de aprendizagem para o processo de desenvolvimento de jogos educacionais ajuda a melhorar o desempenho dos estudantes? Em caso positivo, como é medido o desempenho?}

Em Ahmad et al. (2012), os autores avaliam as teorias de aprendizagem aplicadas para o desenvolvimento do jogo por meio de observações, porém, os conceitos foram utilizados principalmente na idealização. Uma pesquisa foi realizada com 15 crianças, que ficaram animadas com o jogo e concordam que é útil para a aprendizagem de línguas. Para avaliar o jogo proposto em Crow e Parsons (2015), os autores fizeram uso de observações (avaliação qualitativa) em sala de aula e pré e pós-teste (avaliação quantitativa). Os resultados apontaram que a ferramenta é atraente e eficaz, e que os alunos conseguiram aprender com o jogo. As observações qualitativas foram realizadas para testar como a mecânica e o ambiente do jogo afetavam as experiências de aprendizagem. Um estudo piloto com 42 crianças diagnosticadas com ADHD foi realizado em Bul et al. (2015), onde testes foram aplicados com pais e alunos. Os pais ficaram satisfeitos em relação ao jogo e informaram que indicariam para outros pais de crianças com ADHD. 44\% das crianças ficaram motivadas com o jogo, 66\% indicaram que aprenderam algo, $77 \%$ informaram que indicariam para outras crianças com ADHD. Em Piki et al. (2016), os autores não citam por qual processo de avaliação os jogos passaram, mas indicam que os educadores são 
responsáveis por avaliar quais os resultados de aprendizagem alcançados e concluem que os jogos puderam apoiar os métodos tradicionais de ensino e oferecer maneiras divertidas para crianças com deficiência intelectual aprenderem.

Em Nikitakos et al. (2017), é realizada avaliação sumativa com 21 estudantes adultos, através de conversa analítica sobre o jogo e sua relação com a educação marítima. Como o jogo ainda estava na versão alfa os estudantes não puderam jogar. Para avaliar o jogo proposto em Sockolow et al. (2018), foram utilizadas análises quantitativas, qualitativas, métodos mistos e determinação da significância clínica, os autores avaliaram a usabilidade e o impacto do jogo quanto à proposta de assistir a adolescentes sobre comportamentos sexuais de risco. Já a etapa de avaliação proposta por Zhou et al. (2018), consiste em melhorar o jogo e verificar se os objetivos da fase de design foram alcançados. Os autores propõem cinco dimensões de avaliação: desempenho (com relação aos resultados de aprendizagem), usabilidade (capacidade de uso e de aprendizagem do jogo), estado cognitivo (determinar se a aprendizagem obteve sucesso), estado afetivo (emoções do usuário durante o jogo) e interação social (interação do aluno com os demais colegas e o professor). Para validar o jogo proposto, os autores realizaram uma avaliação com 22 estudantes universitários. Os resultados não apresentaram diferenças significativas entre pré (antes de jogar) e pós-teste (depois de jogar), porém, demais testes mostraram uma boa experiência de usabilidade, emoções positivas e um nível adequado de desafio.

Por ser um trabalho em andamento, em Fonseca et al. (2018) não são apresentados resultados de aplicação do jogo, porém eles descrevem o planejamento de avaliação a ser realizada. O jogo será aplicado com alunos do ensino fundamental e médio e a avaliação se dará a partir de pré e pós-teste comparando o resultado de três grupos: experimental 1 (jogará o jogo), experimental 2 (terá uma palestra) e de controle (sem nenhuma intervenção). Já em Mohd Rashid et al. (2019), os autores fizeram um experimento de pré e pós teste com 20 alunos de uma escola primária. Os resultados mostraram que a nota do pós-teste foi superior a do pré-teste, concluindo que existiu um melhora no aprendizagem. Ros et al. (2020), propuseram um modelo de equação estrutural baseado em construtores para avaliar os elementos de design que influenciaram na autopercepção dos alunos quanto ao sucesso na aprendizagem. O resultado apontou para engajamento (capacidade do jogo de capturar a atenção do jogador) e contextualização do jogo. Para verificar a eficácia, foi realizado um experimento de comparação entre dois grupos: jogadores e não-jogadores, de uma turma de Ciência da Computação. Os alunos que decidiram jogar, obtiveram notas mais altas em um contexto geral.

Ao responder essa questão de pesquisa, notou-se que em alguns artigos os autores apresentam resultados positivos com relação ao jogo, mas não informam de que maneira chegaram a essas conclusões e como avaliaram o jogo de fato.

\section{Conclusões}

Esse MSL teve como objetivo identificar as metodologias de desenvolvimento de jogos educacionais e as teorias de aprendizagem aplicadas. A partir dos dez artigos selecionados, foi possível verificar o método escolhido para projetar os jogos e critérios de aprendizagem utilizados, o que respondeu as duas questões secundárias propostas.

A QP1.1 tinha como foco descobrir os processos de desenvolvimento abordados para jogos educacionais. Como discutido na seção anterior, alguns autores se basearam 
em metodologias existentes para criar um processo de desenvolvimento próprio para o seu jogo, outros utilizaram métodos específicos para criação de jogos educacionais como o modelo ADDIE. Também verificou-se o uso de metodologias de desenvolvimento de sistemas e propostas de métodos para projetar jogos educacionais. Um dos objetivos da MSL é identificar a abordagem de teorias e critérios de aprendizagem no processo de desenvolvimento de jogos educacionais (QP1.2). Nos dez artigos selecionados, os autores descrevem sobre a importância de se basear em aspectos teóricos para desenvolver jogos educacionais. Dentre as teorias de aprendizagem, destaca-se o Construtivismo. Alguns estudos, utilizam teorias específicas para o objetivo de aprendizagem, por exemplo, para crianças com deficiência intelectual, a escolha é por teorias psicológicas. A teoria do engajamento também é abordada em um estudo, mas a maioria dos autores discorrem sobre a importância de engajar e motivar os estudantes por meio dos jogos.

A questão principal (QP1) buscou identificar as vantagens da utilização de critérios de aprendizagem no processo de desenvolvimento dos jogos educacionais. Para isso, foram analisados os resultados apresentados quanto à aplicação dos jogos propostos. Avaliações de pré-teste, pós-teste, comparação entre grupos e análise por observação foram as principais formas de analisar os benefícios do jogo para os alunos. Alguns autores não citam o processo de avaliação, mas concluem o potencial do uso de jogos para a educação. Embora seja possível identificar os aspectos desejados para a pesquisa, a avaliação dos jogos, investigada pela questão principal, foi a menos explorada pelos autores dos estudos. Os resultados desse MSL apontam a importância de se basear em teorias de aprendizagem para projetar jogos educacionais. Porém, para analisar se os jogos são eficazes para os estudantes, os pesquisadores devem investir em métodos de avaliação.

\section{Referências}

Ahmad, W. F. W., Shaarani, A. R. S., and Afrizal, S. (2012). Mobile language translation game. In 2012 International Conference on Computer \& Information Science (ICCIS), volume 2, pages 1099-1104. IEEE.

Balasubramanian, N. and Wilson, B. G. (2006). Games and simulations. In Society for information technology and teacher education international conference. Citeseer.

Bul, K. C., Franken, I. H., Van der Oord, S., Kato, P. M., Danckaerts, M., Vreeke, L. J., Willems, A., Van Oers, H. J., Van den Heuvel, R., Van Slagmaat, R., et al. (2015). Development and user satisfaction of "plan-it commander," a serious game for children with adhd. Games for health journal, 4(6):502-512.

Cohen, J. (1960). A coefficient of agreement for nominal scales. Educational and psychological measurement, 20(1):37-46.

Crow, T. and Parsons, D. (2015). A mobile game world for māori language learning. In International Conference on Mobile and Contextual Learning, pages 84-98. Springer.

Fonseca, S., González, S., Rodríguez, B., Seda, M., Bobonis, J., Canela, A., Rolón, C., Bosque, J., Méndez, L., Cartagena, A., et al. (2018). Educational nanotechnology video game to inspire middle and high school students to pursue stem related professional careers. In Frontiers in Education Conference (FIE), pages 1-5. IEEE.

Gattegno, C. (2010). Teaching foreign languages in schools: The silent way. Educational Solutions World. 
IX Congresso Brasileiro de Informática na Educação (CBIE 2020)

Anais do XXXI Simpósio Brasileiro de Informática na Educação (SBIE 2020)

Gros, B. (2003). The impact of digital games in education. First Monday, 8(7):6-26.

Hsiao, H.-C. (2007). A brief review of digital games and learning. In 2007 First IEEE International Workshop on Digital Game and Intelligent Toy Enhanced Learning (DIGITEL'07), pages 124-129. IEEE.

Kattenbelt, C. and Raessens, J. (2003). Computer games and the complexity of experience.

Kitchenham, B. and Charters, S. (2007). Guidelines for performing systematic literature reviews in software engineering. Technical report, Technical report, Ver. 2.3 EBSE Technical Report. EBSE.

Linehan, C., Kirman, B., Lawson, S., and Chan, G. (2011). Practical, appropriate, empirically-validated guidelines for designing educational games. In Proceedings of the SIGCHI conference on human factors in computing systems, pages 1979-1988. ACM.

Mohd Rashid, N. A. B., Salleh, S. B. M., and Noor, N. B. M. (2019). Development of jawi spelling skills applications,'oh jawiku'. International Journal of Interactive Mobile Technologies, 13(7).

Newman, J. (2012). Videogames. Routledge, 2 edition.

Nikitakos, N., Sirris, I., Dalaklis, D., Papachristos, D., and Tsoukalas, V. (2017). Gamebased learning for maritime education and training: the case of trader of the world. WMU Journal of Maritime Affairs, 16(2):265-291.

Oliveira, E., Viana, D., Cristo, M., and Conte, T. (2017). Como os pesquisadores de engenharia de software mediram a produtividade do software? -um estudo de mapeamento sistemático. In Conferência Internacional sobre Sistemas de Informação Empresarial, volume 2, pages 76-87. SCITEPRESS.

Petersen, K., Vakkalanka, S., and Kuzniarz, L. (2015). Guidelines for conducting systematic mapping studies in software engineering: An update. Information and Software Technology, 64:1-18.

Piki, A., Markou, M., and Vasiliou, A. (2016). Learning through play: The role of learning and engagement theory in the development of educational games for intellectually challenged children. In 2016 International Conference on Interactive Technologies and Games (ITAG), pages 1-6. IEEE.

Ros, S., González, S., Robles, A., Tobarra, L., Caminero, A., and Cano, J. (2020). Analyzing students' self-perception of success and learning effectiveness using gamification in an online cybersecurity course. IEEE Access.

Savi, R. and Ulbricht, V. R. (2008). Jogos digitais educacionais: benefícios e desafios. Renote, 6(1).

Sockolow, P., Joppa, M., and Zhu, J. (2018). Assessment of evaluation frameworks for design of a sexual risk prevention game for black adolescent girls. Studies in health technology and informatics, 250:101-105.

Zhou, Y., Xu, T., Zhu, Z., and Wang, Z. (2018). Learning in doing: a model of design and assessment for using new interaction in educational game. In International Conference on Learning and Collaboration Technologies, pages 225-236. Springer. 